\title{
Focused Ion Beam: Much More Than a Sample Preparation Tool
}

\author{
P.E. Russell ${ }^{1}$, T.J. Stark ${ }^{2}$, J.P. Viterelli ${ }^{2}$, A.R. Guichard ${ }^{1}$, J. Wang $^{1}$, K. L. Bunker ${ }^{1}$, J.C. \\ Gonzalez $^{1}$, and D.P. Griffis ${ }^{1}$ \\ ${ }^{1}$ Analytical Instrumentation Facility, North Carolina State University, Raleigh, NC \\ 27502-7531, ${ }^{2}$ Materials Analytical Services, Raleigh, NC 27606
}

The $\mathrm{Ga}^{+}$Focused Ion Beam (FIB) is a very versatile tool which, although established as the preeminent tool for microcircuit edit, has recently evolved into the preferred TEM sample preparation tool for site specific applications. However, the applications of FIB extend far beyond the tasks for which FIB is most commonly employed. FIB can be utilized for micro and nano structural creation and/or modification. This can be accomplished by taking advantage of FIB's capability to very precisely remove material (via physical sputtering or using beam induced chemistry for enhancing material removal rate), to deposit material (using beam induced chemistry), to provide localized ion implantation, and to locally induce sample structural damage. In addition to the above capabilities, FIB provides the ability to image the sample via secondary electrons or ions before, after and during micromachining. In many cases, the ability to image while removing or depositing material provides invaluable feedback for process evaluation and control.

In this presentation, an overview of FIB will be presented with emphasis on non traditional applications. Examples presented will include a wide range of FIB based nano and micro fabrication techniques including stamp/mold fabrication (Fig. 1), shaping of a diamond indenter tool (Fig. 2), AFM tip characterization structure fabrication (Fig. 3), sharpening of an STM tip (Figure 4), MEMS device cross section (Fig. 5), etc.

In some instances where micro or nano material removal or deposition is required, it is possible to perform these tasks using an electron rather than an ion beam. This can be especially advantageous when the Ga "stain" left behind during all FIB processes is deleterious to the desired result e.g. during EUV mask repair. The electron beam when use in conjunction with appropriate chemical precursors, can initiate chemical reactions resulting in either material removal or material deposition. Examples will be presented showing the ability to precisely remove and deposit materials using an electron beam.

In summary, FIB is a versatile tool that combines both nanofabrication and microscopy capabilities. 

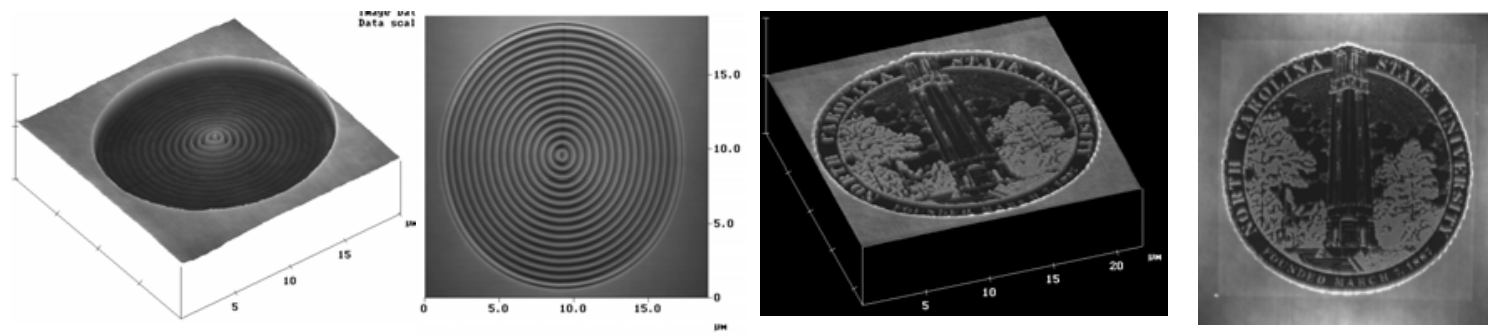

Fig. 1. SEM and AFM images of a micro fabricated Fresnel lens and the NCSU seal. These micro fabricated structures could be used for either stamps or molds.
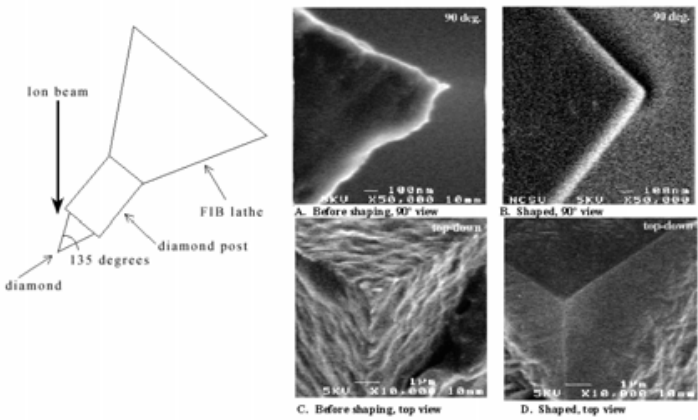

Fig 2. SEM micrographs of a diamond indenter tip before and after shaping using FIB.
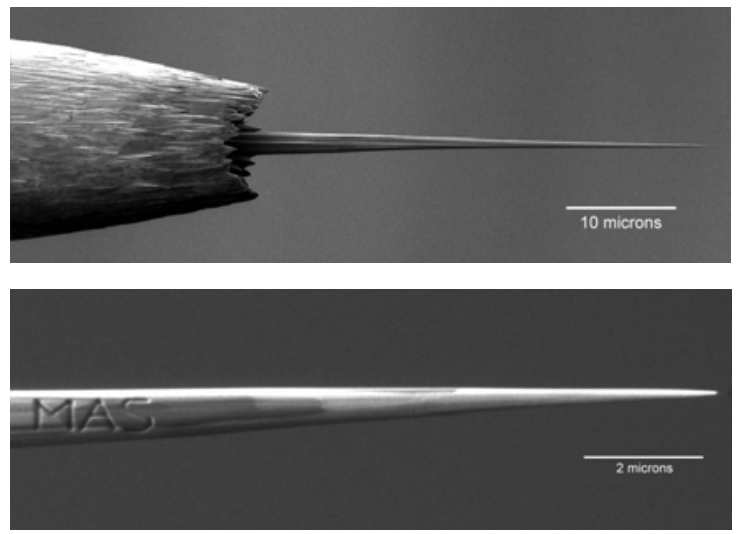

Fig 4. SEM micrographs of an STM tip sharpened using FIG. Note inscription on tip in lower image.

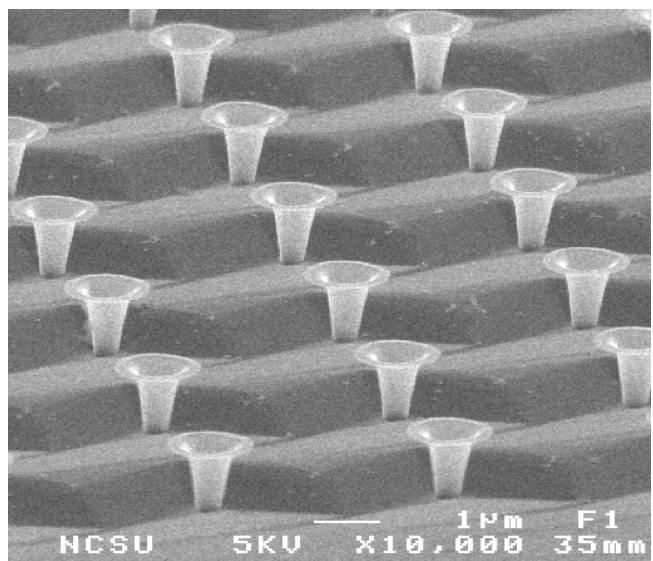

Fig 3. SEM micrographs of a structure fabricated using FIB implantation in Si followed by etching.

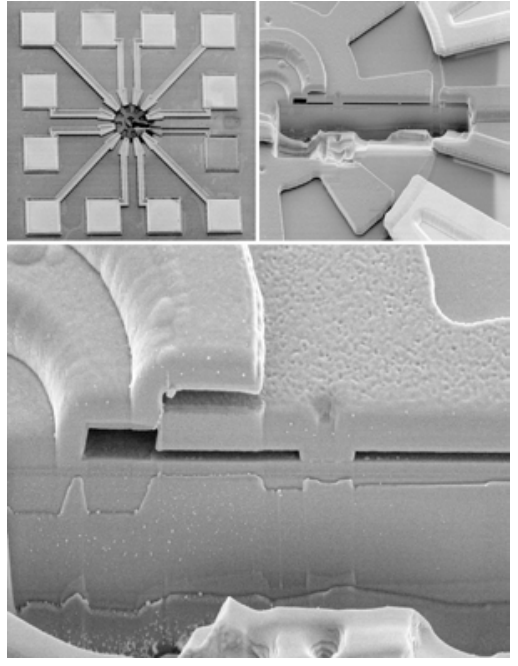

Fig 5. SEM micrographs of a MEMS motor after FIB cross sectioning. 